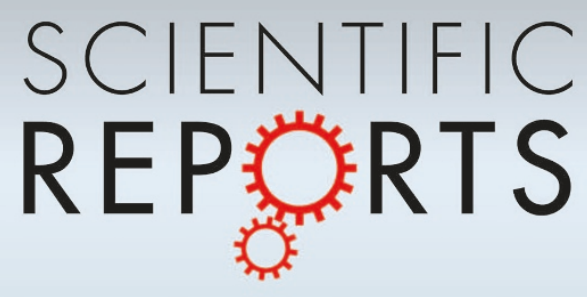

\title{
Fully printed flexible and disposable wireless cyclic voltammetry tag
}

SUBJECT AREAS:

ENVIRONMENTAL MONITORING

ELECTRICAL AND ELECTRONIC ENGINEERING

Received

22 October 2014

Accepted

6 January 2015

Published

29 January 2015

Correspondence and requests for materials should be addressed to G.C. Igcho@sunchon. ac.kr)

\author{
Younsu Jung ' , Hyejin Park', Jin-Ah Park', Jinsoo Noh' ', Yunchang Choi ', Minhoon Jung'2, \\ Kyunghwan Jung ${ }^{2}$, Myungho Pyo', Kevin Chen ${ }^{3}$, Ali Javey ${ }^{3} \&$ Gyoujin Cho'
}

\begin{abstract}
'Department of Printed Electronics Engineering, Sunchon National University, Maegok, Sunchon, Jeonnam 540-742 Korea, ${ }^{2}$ Research Institute of Printed Electronics, PARU Co. Seomyeon, Sunchon, Jeonnam 540-813 Korea, ${ }^{3}$ Electrical Engineering and Computer Sciences, University of California, Berkeley, CA 94720.
\end{abstract}

A disposable cyclic voltammetry (CV) tag is printed on a plastic film by integrating wireless power transmitter, polarized triangle wave generator, electrochemical cell and signage through a scalable gravure printing method. By proximity of $13.56 \mathrm{MHz} \mathrm{RF}$ reader, the printed CV tag generates $320 \mathrm{mHz}$ of triangular sweep wave from $+500 \mathrm{mV}$ to $-500 \mathrm{mV}$ which enable to scan a printed electrochemical cell in the CV tag. By simply dropping any specimen solution on the electrochemical cell in the CV tag, the presence of solutes in the solution can be detected and shown on the signage of the CV tag in five sec. $10 \mathrm{mM}$ of $\mathrm{N}, \mathrm{N}, \mathrm{N}^{\prime}, \mathrm{N}^{\prime}$-tetramethyl-p-phenylenediamine (TMPD) was used as a standard solute to prove the working concept of fully printed disposable wireless $\mathrm{CV}$ tag. Within five seconds, we can wirelessly diagnose the presence of TMPD in the solution using the $\mathrm{CV}$ tag in the proximity of the $13.56 \mathrm{MHz} R F$ reader. This fully printed and wirelessly operated flexible CV tag is the first of its kind and marks the path for the utilization of inexpensive and disposable wireless electrochemical sensor systems for initial diagnose hazardous chemicals and biological molecules to improve public hygiene and health.

yclic voltammetry (CV) has been used as a powerful tool for the study of electrochemical redox reactions between the electrodes and the solutions of all kind of solutes such as metals ${ }^{1}$, organic molecules ${ }^{2}$, proteins $^{3}$, bacteria ${ }^{4}$, viruses ${ }^{5}$, and $\mathrm{DNA}^{6}$. Because of the high sensitivity of the electron transfer redox reactions, CV could become a very promising ubiquitous electrochemical sensor protocol if the cost and size of the $\mathrm{CV}$ system were significantly reduced to commercially viable single-use disposable units for checking traces of hazardous materials such as lead ${ }^{7}$, mercury $^{8}$, arsenic ${ }^{9}$, e-coli $^{10}$, and pesticides ${ }^{11}$ in water or to diagnose the level of glucose $^{12}$, cholesterol ${ }^{13}$ and specific enzymes ${ }^{14}$ in blood. This single-use disposable CV would dramatically reduce the cost of maintaining the public health system ${ }^{15}$. Therefore, inexpensive and disposable CV measurement system that can be operated wirelessly using a smartphone or RF (radio frequency) reader without complicate operation processes is in high demand for the realization of an ubiquitous sensor network system (Figure 1a). They would mainly be used as ubiquitous diagnostic and testing tools for detecting and monitoring the level of target specimens. However, there is no technology that is advanced enough yet to build a wireless, inexpensive and disposable CV system. In this paper, as a form of RF-tag, an extremely inexpensive, disposable and fully printed $\mathrm{CV}$ system is demonstrated for the first time by mimicking and combining basic CV concepts and wireless power transmission technologies of RF devices. To realize the fully printed CV tag, a key issue of wirelessly generating triangular waveform $( \pm 500 \mathrm{mV})$ that can scan the electrochemical cell at a low frequency $(<1 \mathrm{~Hz})$ to set up a redox reaction needs to be addressed through a minimum number of printed thin film transistors (TFTs). Fully printed $13.56 \mathrm{MHz}$ rectenna and a ring oscillator with large trap charges in the channels of printed TFTs were respectively utilized to wirelessly generate triangular waveform by using only 10 printed TFTs.

\section{Results}

Based on two key units of the wireless power transmitter (Figure 1b-1) and the triangular wave generator (Figure 1b-2), the circuit layout of the fully printed wireless CV tag was designed by using a minimum number of printed thin film transistors (TFTs) to alleviate the issue of $\mathrm{V}_{\mathrm{th}}$ shift and shown in Figure $1 \mathrm{~b}$ as a platform of disposable CV system. The circuit of CV tag was fabricated using all scalable printing methods, a roll-to-roll gravure, a roll-to-plate gravure, a drop casting, and a screen printer (Figure S1 in Supplementary Information).

To provide a polarized DC voltage from the coupled $13.56 \mathrm{MHz}$ AC signal of the reader, we modified our previously reported R2R gravure printed rectenna ${ }^{16,17}$, as shown in the layout in Figure 2a and the resulting 
a

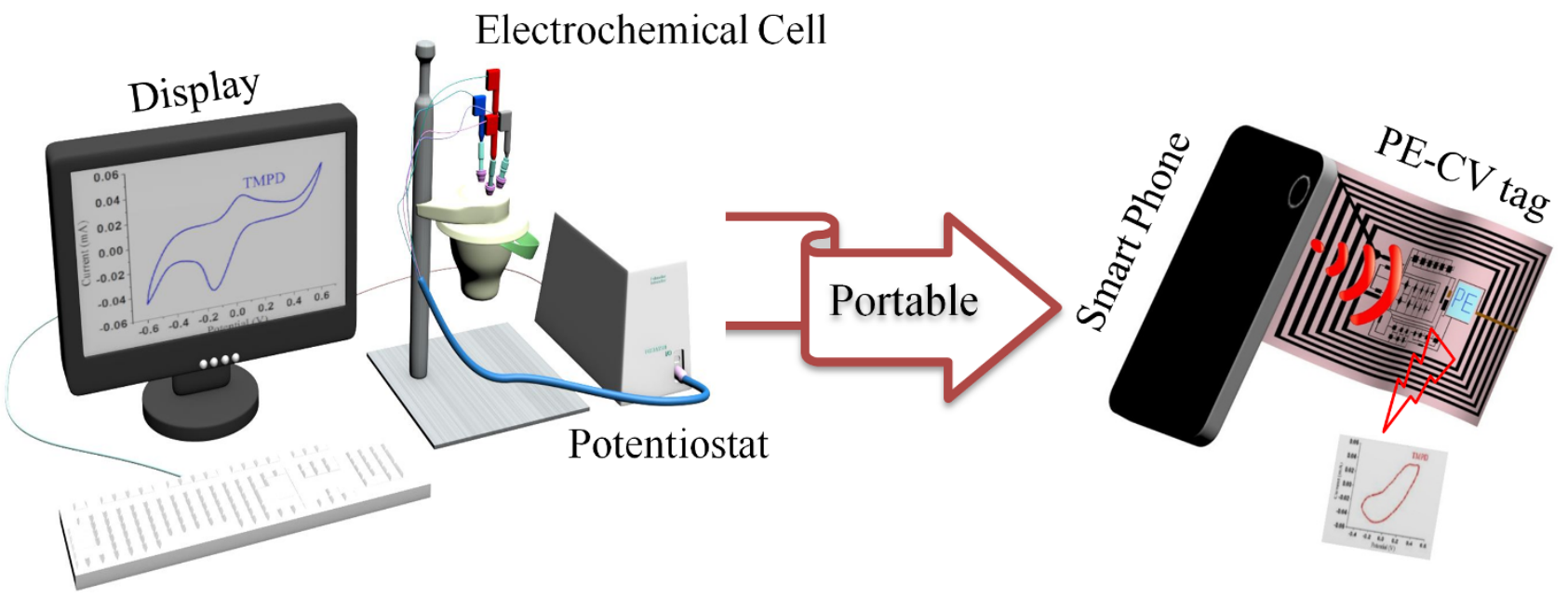

b

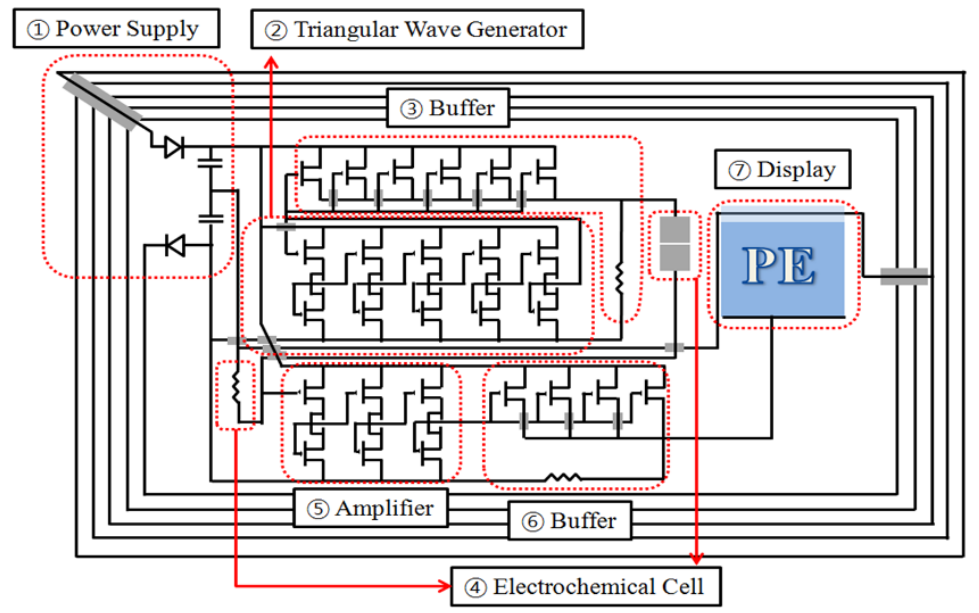

Figure 1 (a) Informative illustration of typical CV system and disposable printed CV tag. (b) Schematic circuit diagram of the gravure printed wireless cyclic voltammetry (CV) tags. The circuit was designed to couple AC power from a $13.56 \mathrm{MHz}$ reader and then convert the coupled AC to polarized $\mathrm{DC} \pm 10 \mathrm{~V}$ (1) in Figure 1b). Polarized DC will operate the printed ring oscillator to generate a triangular voltage waveform (2) in Figure 1b). The generated waveform will pass through the buffer to meet the impedance difference from the electrochemical cell (3) in Figure 1b). The electrochemical cell will run the redox reaction with a single drop of specimen solution (4) in Figure 1b) by the voltage triangular waveform. The output current of the electrochemical redox reaction will be amplified via an amplifier circuit (5) in Figure b) and the signal will pass through the buffer to meet the impedance difference from the printed signage (6) in Figure b). The signage will indicate the concentration level of specimen in the solution (7) in Figure b). It will indicate whether the concentration is above or below a pre-determined value.

printed diodes and capacitors are shown in Figure $2 \mathrm{~b}$. The printed diodes showed a rectifying ratio of $10^{3}$, and capacitances of two printed capacitors were all about $8 \mathrm{nF} / \mathrm{cm}^{2}$. The polarized DC voltages (Figure $2 \mathrm{c}$ ) were measured by placing the printed rectenna on the RF $(13.56 \mathrm{MHz})$ reader with a distance of $2 \mathrm{~cm}$. We adapted a center tap transformer, consisted by divided antenna, 2 diodes and 2 capacitors, to provide the + and - DC voltage for printed triangle wave generator. Using a load of $1 \mathrm{M} \Omega$, voltages of $+9.4 \mathrm{~V}$ and $-10.8 \mathrm{~V} \mathrm{DC}$ were attained for the optimized printed antenna by monitoring the rectified polarized DC voltages and varying the values of the inductance of the antenna (Figure S2 in Supplementary Information). The polarized DC voltage was also decreased with decreasing load's resistance (Figure $2 \mathrm{~d}$ ).

To generate a cyclic waveform with a low frequency $(<1 \mathrm{~Hz})$ from the rectified polarized DC voltage, fully gravure printed $p$-type SWNT-based channel network thin film transistors ( $c n$ TFTs) were used to construct a five stage ring oscillator with $p$-type inverters. The $c n$ TFTs can operate under less than $20 \mathrm{~V}$ and their mobility range $\left(0.01\right.$ to $\left.10 \mathrm{~cm}^{2} / \mathrm{Vs}\right)$ can be controlled through the loading concentration of SWNT in the ink ${ }^{18-21}$. To print the five stage ring oscillator, we used the same silver and $\mathrm{BaTiO}_{3}$ inks as in the printing of the rectenna for reducing a number of printing steps while SWNT ink was used for printing the channel of the $c n$ TFTs. To manufacture the ring oscillator, roll-to-plate (R2P) gravure was used to print 10 cnTFTs with a gate width of $300 \mu \mathrm{m}$ and channel length of $160 \mu \mathrm{m}$ for both the drive and load $c n$ TFTs (Figure S3 in Supplementary Information). The optimized cell depth of gravure plate was 37$40 \mu \mathrm{m}$ with the cell wall thickness of $50-55 \mu \mathrm{m}$ for the printing active layers of drive and load TFTs using low viscosity of SWNT based ink ( $\sim 30 \mathrm{cp}$ ) (Figure S4 in Supplementary Information). By repeating the printing of active layers on load TFTs, a higher SWNT network density can be achieved to generate higher current in the 
a

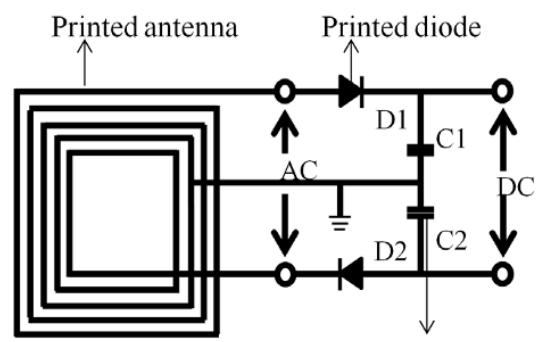

Printed capacitance

C

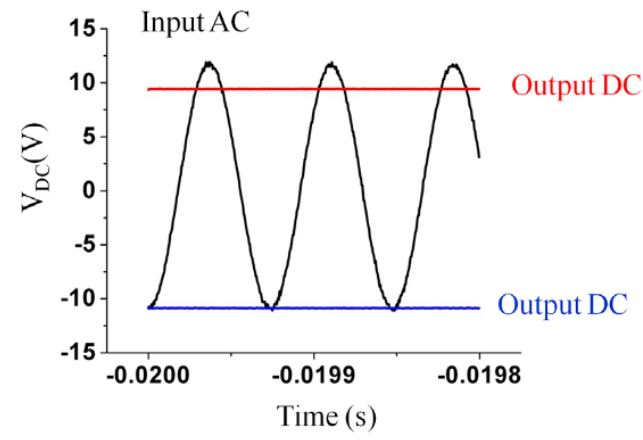

b

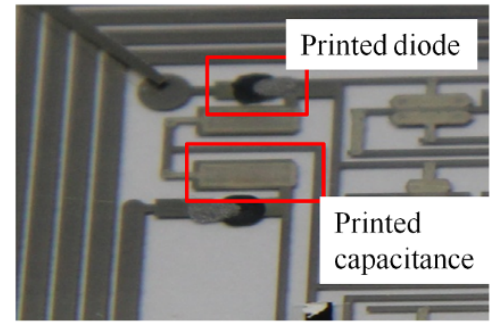

d

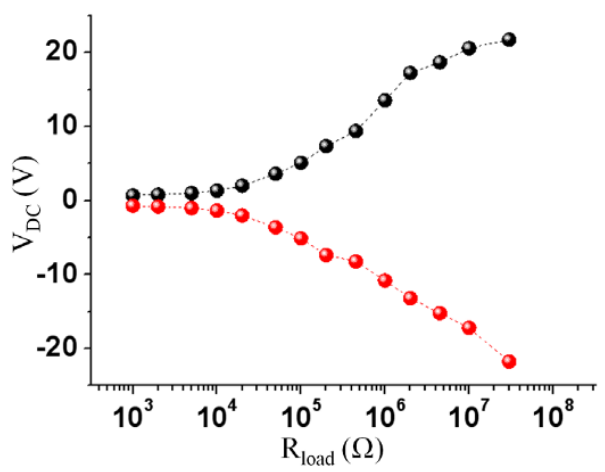

Figure $2 \mid$ (a) Circuit layout of the printed rectenna to provide polarized DC power, (b) a real image of printed diodes and capacitors, (c) input-output electrical characteristics of the rectifier at $13.56 \mathrm{MHz}$ AC input and (d) output \pm DC voltages under variations in load resistance.

channels while thicknesses of all printed gate, dielectric and drainsource electrodes are kept same (Figure S5 in Supplementary Information).

Their output and transfer characteristics are shown in Figure 3a and $3 \mathrm{~b}$ respectively. Based on the attained transfer characteristics, the mobility $(\mu)$, transconductance $\left(g_{m}\right)$, on-off current ratios from $\mathrm{I}_{\mathrm{DS}^{-}}$ $\mathrm{V}_{\mathrm{DS}}$ and threshold voltages $\left(\mathrm{V}_{\mathrm{th}}\right)$ for both five drive cnTFTs and five load $c n$ TFTs were extracted and shown in Table S1 (Supplementary Information). The shift range of $\mathrm{V}_{\text {th }}$ from the fully gravure printed 10 $c n$ TFTs were about $\pm 0.4 \mathrm{~V}$ for the drive $c n$ TFTs and $\pm 0.6 \mathrm{~V}$ for load cnTFTs. Those shift ranges are narrow enough to run the printed ring oscillator for generating the triangular wave. Each inverter delivered a gain of 3 (Figure 3c), and the ring oscillator with five inverters generated a pseudo triangular waveform with a voltage amplitude of $7 \mathrm{~V}$ at $320 \mathrm{mHz}$ (Figure 3d). In fact, the pseudo triangular wave can be only generated by the printed $c n$ TFTs based ring oscillator because of the parasitic and trapped channel capacitances due to carbon nanotube network structures in cnTFTs. Although we only showed the frequency of $320 \mathrm{mHz}$ at here, the frequency can be varied from $0.3 \mathrm{~Hz}$ to $2 \mathrm{~Hz}$ based on the printed network density of SWNT of printed cnTFTs. This is an advantage of our printed cnTFTs for scanning electrochemical cell in CV tag over other technologies such as amorphous silicon TFTs where only a sine wave with a designed single frequency can be generated because of no trap capacitance in the channel (Figure S6 in Supplementary Information). The frequency of the triangular waveform can be fine-tuned in the range of $0.3 \mathrm{~Hz}$ to $2 \mathrm{~Hz}$ as shown in the Figure S7 (Supplementary Information) by simply changing loading concentration of SWNTs.

When the generated triangular waveform is supplied directly into the printed electro-chemical cell (Figure S8 in Supplementary Information), wherein printed silver and carbon electrodes were used with a poly(ethylene oxide) and $\mathrm{LiCF}_{3} \mathrm{SO}_{3}$ gel type electrolyte, the output voltage range is reduced to zero because of improper impedance matching between the electrochemical cell $(10 \mathrm{~K} \Omega)$ and ring oscillator (1 M $\Omega$ ). Therefore, a buffer unit is needed to match the impedance levels between the ring oscillator and electrochemical cell. The printed buffer unit (Figure 1b-(3) with high on currents and low on-off current ratios (Figure 3e) is consists of $6 \mathrm{cnTFTs}$ and a resistor $(\sim 7 \mathrm{~K} \Omega)$. In the printed $\mathrm{CV}$ tag, three resistors were printed by screen printer using a carbon paste (DC-20, purchased from Dozen Co. Korea) for the buffer units (Figure 1b-(3) and (6) and the electrochemical cell (Figure 1b-(4)). Their electrical parameters are listed in Table S1 (Supplementary Information). The 6 cnTFTs of buffer unit were printed using R2P gravure with high SWNT concentration in the semiconducting ink. Each cnTFTs exhibited oncurrents in the range of $400 \sim 500 \mu \mathrm{A}$ with a very low on-off ratio due to the enhanced metallic percolation in the dense SWNT networks. The buffer units with low on-off ratio were employed to reduce the input voltage to $\pm 500 \mathrm{mV}$ (Figure $3 \mathrm{f}$ ) which is the appropriate scanning range of the electrochemical cell. The printed electrochemical cell with two electrodes was proven to be well operated under commercial CV (Figure S9 in Supplementary Information). The resulting current after scanning printed electrochemical cell in the CV tag can be converted to voltage by connecting the printed resistor (Figure 1b-(4): $\sim 510^{3} \mathrm{ohm}$ ) which provides the output voltage for electrochromic indicator and re-plotted into the cyclic voltammogram.

Because these output voltage $( \pm 70 \mathrm{mV})$ and current $(\sim 40 \mu \mathrm{A})$ were low after scanning the electrochemical cell, they need to be amplified to turn on the electrochromic indicator for showing predetermined concentration (10 mM) level of $\mathrm{N}, \mathrm{N}, \mathrm{N}^{\prime}, \mathrm{N}^{\prime}$-tetramethylp-phenylenediamine (TMPD, purchased from Aldrich) as a standard reference in this work (Figure $3 \mathrm{~g}$ ). After running the redox reaction of TMPD (Figure $3 \mathrm{~h}$ ) in the printed cell, the output voltage and current needed to be amplified to reach $1.5 \mathrm{~V}$ and $500 \mu \mathrm{A}$, respectively, to provide sufficient power to display the letters "PE" as an indicator of the presence of TMPD in the specimen. To construct the amplifier, cnTFTs based three inverters were printed with a gain of three to five (Figure S10 in Supplementary Information) to amplify the output signal as shown in Figure 3i. The electrical characteristics of the $6 \mathrm{cnTFTs}$ in the inverters with extracted mobility, transcon- 


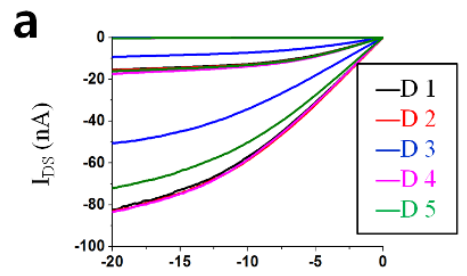

$\mathrm{V}_{\mathrm{DS}}(\mathrm{V})$ for Drive TFT

\section{C}

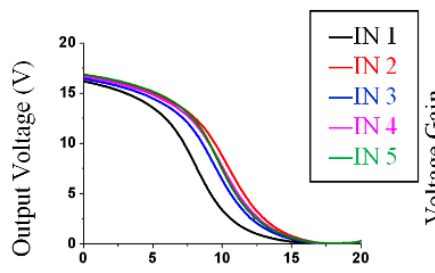

Input Voltage (V)

f

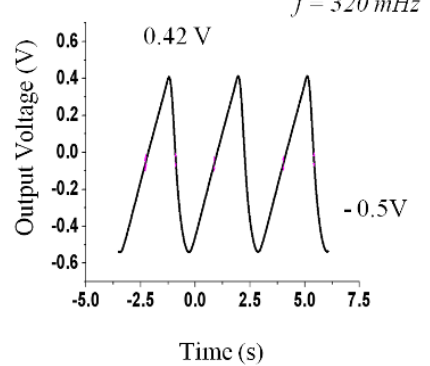

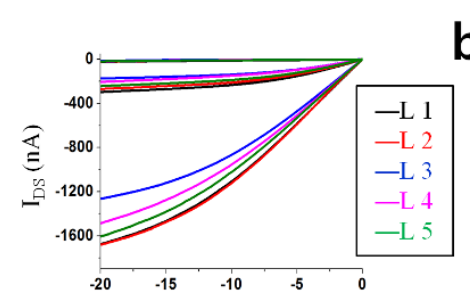

$\mathrm{V}_{\mathrm{DS}}(\mathrm{V})$ for Load TFT b

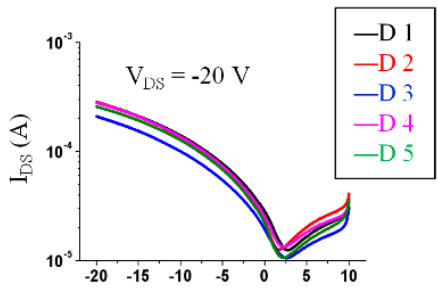

$\mathrm{V}_{\mathrm{GS}}(\mathrm{V})$ for Drive TFT

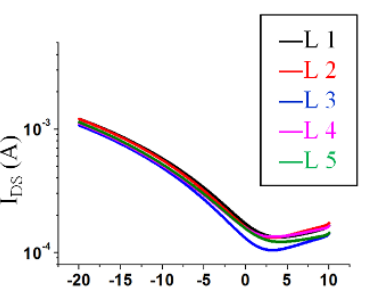

$\mathrm{V}_{\mathrm{GS}}(\mathrm{V})$ for Load TFT d

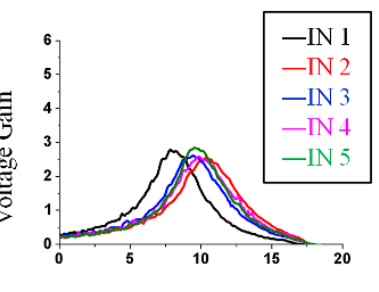

Input Voltage (V)

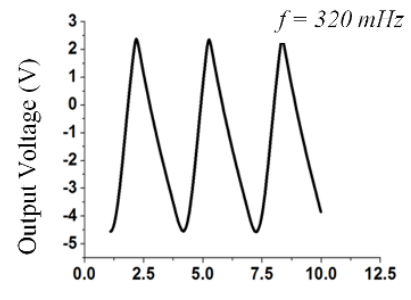

Time (s) e

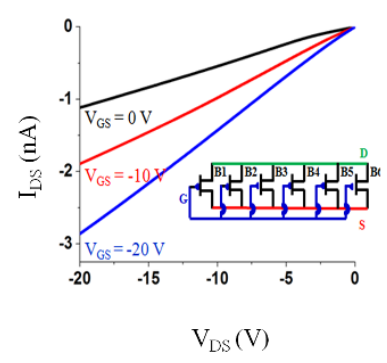

TMPD

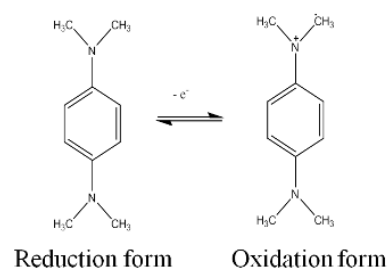

i

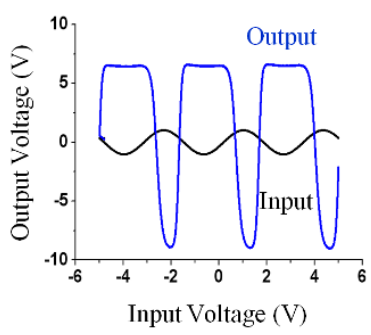

Figure $3 \mid$ ( $\mathrm{a}$ and $\mathrm{b}$ ) Output and transfer characteristics of the printed $c n$ TFTs for 5 drive and 5 load TFTs respectively in the printed ring oscillator. (c) Electrical characteristics of inverters and (d) output characteristic of the ring oscillator. (e) Total output characteristics of the printed buffer unit consisting of $6 \mathrm{cnTFTs}$ and a resistor (measured based on contacting gate and drain-source electrodes as shown in the inset circuit). (f) Modified triangular wave following the buffer unit. (g) Generated signals before scanning (black) and after scanning the electrochemical cells without (1, blue) and with TMPD (2, red) in a drop of solution. (h) TMPD structure for oxidation and reduction reaction. (i) The input and amplified output signals after passing through three amplifying inverters.

ductance, on-off current ratios and threshold voltage are shown in Table S2 (Supplementary Information). As the impedance was matched between the ring oscillator and the electrochemical cell using the buffer unit, another buffer unit (Figure 1b-(6)) is also needed between the electrochromic signage $(20 \mathrm{~K} \Omega)$ and the amplifier (1 M $\Omega$ ) for impedance matching. The electrical parameters of the buffer are listed in Table S2 (Supplementary Information).

The amplified output signal was used to run the reduction and oxidation of the patterned conducting polymer in the electrochromic signage, and thus the blinking signage will be used to indicate the presence of chemicals in the specimens. The signage was fully printed and attached on printed CV tag. A clear concept of the printing sequence for the electrochromic signage was given in Figure S11 in Supplementary Information.

\section{Discussion}

The fully printed flexible CV tag was completed by assembling the printed circuits including ring oscillator, buffer, electrochemical cell, amplifier, and signage onto the previously R2R gravure printed rectenna. The resultant CV tag is shown in Figure 4a. The working concept of the wireless and flexible $\mathrm{CV}$ tag is demonstrated in the following sequences (watch the video file by clicking Figure S12 in Supplementary Information). After dropping $500 \mu \mathrm{l}$ of TMPD solu- tion $(10 \mathrm{mM})$ on the printed electrochemical cell, the CV tag was placed on the custom made RF $(13.56 \mathrm{MHz})$ reader (Figure S13 in Supplementary Information). The antenna was subsequently coupled to $13.56 \mathrm{MHz}$ AC. The coupled AC was rectified into polarized DC $(> \pm 10 \mathrm{~V})$ through two diodes and two capacitors which caused the ring oscillator to generate a pseudo triangular waveform with output voltage of $7 \mathrm{~V}$ at $320 \mathrm{mHz}$. This was then passed through the buffer unit to scan the electrochemical cell. The output signal after scanning the electrochemical cell was amplified to turn on the signage according to the concentration level of TMPD in the solution. In this work, whenever the concentration of TMPD was higher than $10 \mathrm{mM}$, the signage "PE" blinked (Figure 4b) while it did not show anything (Figure 4c) when it was lower than $10 \mathrm{mM}$. Furthermore, clear cyclic voltammograms for scanning the electrochemical cell with and without $10 \mathrm{mM}$ of TMPD were obtained by re-plotting the output triangular voltage waveform (Figure $4 \mathrm{~d}$ ). The attained half potential $\left(\mathrm{E}_{1 / 2} \sim 0.05 \mathrm{~V}\right.$, oxidation potential is $0.22 \mathrm{~V}$ and reduction potential is $-0.17 \mathrm{~V}$ ) of TMPD from the $\mathrm{CV}$ tag was nearly identical to the value obtained from a commercial CV instrument, SP-240, Biologic, which uses the same frequency for the triangular voltage waveform and printed electrochemical cell (Figure 4e). However, when the generated triangle wave frequency is higher than $0.6 \mathrm{~Hz}$ in the $\mathrm{CV}$ tag, the clear redox peaks cannot be 

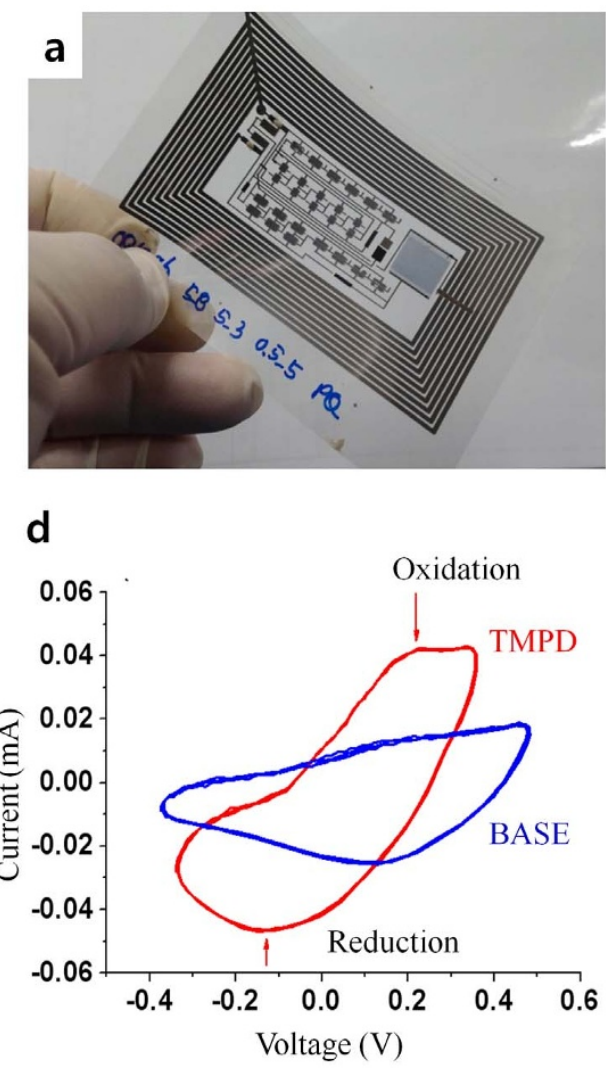

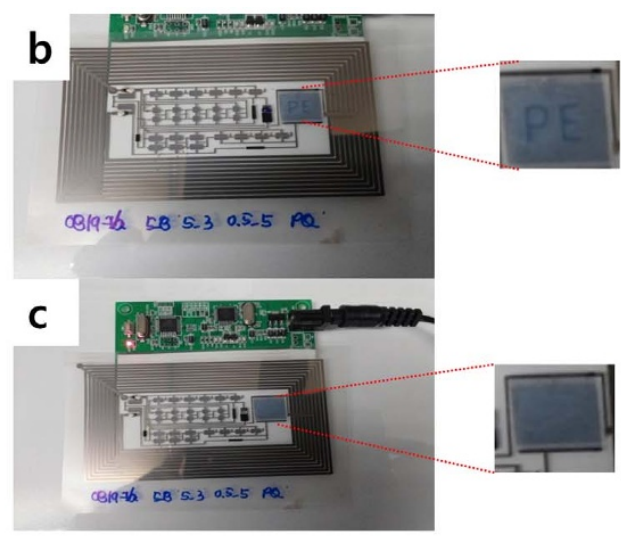

e

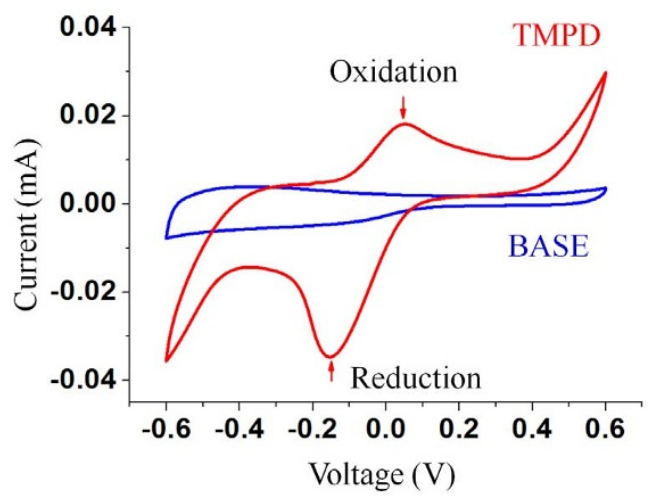

Figure 4| (a) Optical image of fully printed wireless cyclic voltammetry tag. (b) Operation images of CV tag with and (c) without TMPD in the solution on 13.56 MHz reader. (d) Converted cyclic voltammogram from the printed wireless CV tag vs (e) a commercial CV instrument (please refer the interconnected video file for the demonstration of wireless CV tag operation in Figure S12 in Supplementary Information).

observed as shown in Figure S14 (Supplementary Information). Furthermore, as a typical example of testing a specimen in aqueous solution, $10 \mathrm{mM}$ of $\mathrm{K}_{3}(\mathrm{FeCN})_{6}$ aqueous solution was checked using the $\mathrm{CV}$ tag, and results of converted cyclic voltammogram was shown in Figure S15 (Supplementary Information). Those results support that the $\mathrm{CV}$ tag can examine specimens not only in organic solution, but also in aqueous one.

Up to present, there has been no commercial success in fully printed TFT-based electronic devices due to some kind of difficulties in integrating a number of printed TFTs through a scalable printing method. As increasing a number of integrated TFTs, the range of threshold voltage shits of TFTs needs to be controlled as narrow as possible to properly operate designed function of printed devices. However, the scalable printing method is not fully understood to integrate a number of TFTs on plastic foils while keeping the constant range of threshold voltage shifts because of difficulty in controlling a constant charge transport through the printed channels. Therefore, the printed TFT-based devices should be constructed by using a minimum number of printed TFTs while a function of printed device needs to be competitive over Si based one. Since the fully printed CV tag required the integration of only $26 \mathrm{cnTFTs}$, the acceptable range of threshold voltage shits of $c n$ TFTs is controllable and achievable by using a scalable printing method such as a roll-toroll gravure. Furthermore, the unique function of printed $\mathrm{CV}$ tags cannot be simply reproduced using a Si technology with a comparable cost to the R2R printing process. Therefore, the fully printed $\mathrm{CV}$ tag will be the first leading model to show a way of how printed TFTbased electronic devices should go for the successful launching in the IT markets.

In the core of the printed CV tag, fully gravure-printed $c n$ TFTs were employed for constructing a circuit consisting of a ring oscillator to generate triangular waveforms, buffer units for an impedance matching, and an amplifier for enhancing output signal to turn on the signage. Those simple units can be combined with a variety of printable sensors and RF devices to create novel devices with an extremely low cost. In other words, the printed rectenna, ring oscillator, buffer and amplifier units in the printed CV tag will be key elements for the construction of variety of printed RF-sensors, this $\mathrm{CV}$ tag will be used as a platform for the further construction of a variety of RF based electrochemical sensors.

In conclusion, we have presented a fully printed flexible CV tag with the size of $9.5 \times 5.5 \mathrm{~cm}^{2}$ that can operate wirelessly using a 13.56 MHz RF reader. However, for the practical purpose, the size of the $\mathrm{CV}$ tag can be reduced up to the half $\left(4 \times 2 \mathrm{~cm}^{2}\right)$ of the current one by optimizing spaces between $c n$ TFTs in the circuit of the CV tag and reducing the operation power to less than DC $5 \mathrm{~V}$ as the current size of $13.56 \mathrm{MHz}$ antenna is designed to provide maximum coupled AC power using a sufficient length of printed antenna pattern (higher inductance). Furthermore, although we utilized R2P gravure, screen printer and drop casting method for the convenience of fabricating the $\mathrm{CV}$ tag in the Lab, the whole fabricating process can be repeated by R2R gravure because the rectenna, ring oscillator, buffers, electrochemical cell, amplifier and signage in the CV tag were all designed to compromise the limit $( \pm 20 \mu \mathrm{m})$ of a registration accuracy of a current R2R gravure system. Therefore, this technology could be used as a platform for disposable wireless electrochemical sensors to a variety of specimens in aqueous and organic solutions to monitor or diagnose pathogens and hazardous materials by modifying the electrodes of the electrochemical cells.

\section{Methods}

Roll-to-roll (R2R) gravure printed antenna, bottom electrodes, gate electrodes and wires. Two color units of R2R gravure system (Taejin Co. Korea: Figure S1 in the Supplementary Information) was employed to print antenna, bottom electrodes and wires on a roll of poly(ethylene terephthalate) (PET) film (width of $200 \mathrm{~mm}$ and 
thickness of $100 \mu \mathrm{m}$, purchased from SKC, Korea) with silver nanoparticle based conducting ink (PG-007 BB type, Paru Co. Korea) and the web transfer speed of $8 \mathrm{~m} / \mathrm{min}$ under roll pressure of $0.5 \mathrm{MPa}$ and web tension of $60 \mathrm{~N}$. The silver ink was further formulated to meet the viscosity of $500 \mathrm{cp}$ and surface tension of $47 \mathrm{mN} / \mathrm{m}$ using respectively ethylene glycol (Aldrich) and Dipropylene glycol methyl ether (Aldrich). The curing time of printed silver layer was $10 \mathrm{sec}$ by passing through a heating chamber of $150^{\circ} \mathrm{C}$, installed in R2R gravure system. The resulting printed silver patterns on PET roll showed the thickness of $450( \pm 50) \mathrm{nm}$ without any defects and were shown in Figure S16a (Supplementary Information)

Roll-to-plate (R2P) gravure printed ring oscillator, buffer, and amplifier. Full R2P gravure system (Figure S1 in the Supplementary Information) was employed to print single walled carbon nanotube network based thin film transistors ( $c n$ TFTs), used as a building block to construct all the integrated circuit of printed cyclic voltammetry (CV) tag (Figure 1). $26 \mathrm{cnTFTs}$ for the integration of circuit in printed CV tag were printed on the previously R2R gravure printed film (printed antenna, bottom electrodes and wires with thickness of $450 \mathrm{~nm}$; Figure S16b in Supplementary Information) following printing sequences. First, $\mathrm{BaTiO}_{3}$ nanoparticle based dielectric ink (PD-100, Paru Co., Korea) was further formulated to meet viscosity $(75 \mathrm{cP})$ and surface tension $(31.8 \mathrm{mN} / \mathrm{m})$ using the ethyl 2-cynoacrylate (Aldrich) As shown in R2P gravure printing scheme (Figure S17 in Supplementary Information), the homogeneous and defect free dielectric layers were first R2P gravure printed with a printing speed of $200 \mathrm{~mm} / \mathrm{s}$ and roll pressure $7.5 \mathrm{~kg}_{\mathrm{f}}$ on previously printed gate electrodes and bottom electrodes (for capacitors) with a thickness of $2.3( \pm 0.1) \mu \mathrm{m}$ and width of $940( \pm 2) \mu \mathrm{m}$ (Figure S5 in Supplementary Information). The printed dielectric layers were cured for $1 \mathrm{~min} 20 \mathrm{sec}$ at $150^{\circ} \mathrm{C}$. Second, single walled carbon nanotube (SWNT) based semiconducting ink (PR-040, Paru Co., Korea) was diluted by diethylene glycol monobutyl ether (Daejung Co., Korea) to $1: 1$ volume ratio for printing active layers using R2P gravure on the printed dielectric layers with a printing speed of $350 \mathrm{~mm} / \mathrm{s}$ and a roll pressure of $4 \mathrm{~kg}_{\mathrm{f}}$. The viscosity and surface tension of diluted SWNT ink were respectively $30 \mathrm{cP}$ and $29 \mathrm{mN} / \mathrm{m}$. The resulting SWNT printed films were dried $1 \mathrm{~min}$ at $150^{\circ} \mathrm{C}$. For using cnTFTs in the buffer unit, we used the dilution volume ratio of $5: 1$ between SWNT ink (PR-040, Paru Co., Korea) and diethylene glycol monobutyl ether for printing only on the TFTs in the buffer unit to increase SWNT network density for providing the high current (Figure S5 in Supplementary Information). Finally, drain-source electrodes were R2P gravure printed using silver nanoparticle based ink (PG-007 AA type, Paru Co., Korea) on SWNT printed film with a viscosity of $800 \mathrm{cP}$ and a surface tension of $42 \mathrm{mN} / \mathrm{m}$. The R2P gravure printing was carried out under a printing speed of $200 \mathrm{~mm} / \mathrm{s}$ and a role pressure of $5 \mathrm{~kg}_{\mathrm{f}}$

Diode fabrication. Although Shottky diodes were able to print using R2R gravure based on our previous reported process ${ }^{16,17}$, the drop casting method was used for the convenience in this work. First, dropping $\mathrm{ZnO}$ based hybrid semiconducting ink (PD070, Paru Co., Korea) on printed silver electrode and then, Al ink (PA-009, Paru Co., Korea) was dropped on the top of $\mathrm{ZnO}$ ink. The resulting diodes were further cured for $5 \mathrm{~min}$ at $120^{\circ} \mathrm{C}$.

Measurements. Formulated and diluted inks were characterized using DCAT21 (Dataphysics Co., Germany) and SV-10 Vibro viscometer (AND Co., Japan) for respectively measure surface tension and viscosity. All measurements in this work were carried out under ambient condition if there are no other comments. Resistance of printed antenna, bottom electrodes, gate electrodes and wires were measured using two probe methods with HP3457A multimeter. Both surface morphology and thickness of printed layers was characterized using surface profiler (NV-2200, Nanosystem, Korea). LCR meter (E4980A, Agilent, USA), semiconductor parameter analyzer (4155C, Agilent, USA) and digital phosphor oscilloscope (DPO 4054, Tektronix, USA) were respectively used to characterize printed $c n$ TFT based circuits.

1. Sanderson, M. D., Kamplain, J. K. \& Bielawski, C. W. Quinone-annulated Nheterocyclic carbene-transition-metal complexes: Observation of $\pi$-back bonding using FT-IR spectroscopy and cyclic voltammetry. J. Am. Chem. Soc 128, 16514-16515 (2006)

2. Lai, R. Y., Fabrizio, E. F., Lu, L., Jenekhe, S. A. \& Bard, A. J. Synthesis, cyclic voltammetrics studies, and electrogenerated chemiluminescence of a new donoracceptor molecule: 3,7-[Bis[4-phenyl-2-quinolyl]]-10-methylphenothiazine. J. Am. Chem. Soc 123, 9112-9118 (2001).

3. Armstrong, F. A., Hill, H. A. \& Walton, N. J. Direct electrochemistry of redox proteins. Acc. Chem. Res 21, 407-413 (1998).

4. Norouzi, P., Ganjali, M. R., Daneshgar, P., Alizadeh, T. \& Mohammadi, A. Development of fast Fourier transformation continuous cyclic voltammetry as a highly sensitive detection system for ultra-trace monitoring of penicillin V. Anal. Biochem 360. 175-184 (2007).

5. Koyama, J. et al. Correlation of redox potentials and inhibitory effects on EpsteinBarr virus activation of 2-azaanthraquinones. Cancer Letters 212, 1-6 (2004).
6. Teijeiro, C., Perez, P., Marin, D. \& Paleček, E. Cyclic voltammetry of mitomycin C and DNA. Bioelectrochemistry and Bioenergetics 38, 77-83 (1995).

7. Zerihun, T. \& Gründler, P. Electrically heated cylindrical microelectrodes. Determination of lead on Pt by cyclic voltammetry and cathodic stripping analysis. J. Electroanal Chem 415, 85-88 (1996).

8. Bond, A. M. \& Scholz, F. Electrochemical, Thermodynamic, and mechanistic data derived from voltammetric studies on insoluble metallocenes, mercury halide and sulfide compounds, mixed silver halide crystals, and other metal complexes following their mechanical transfer to a graphite electrode. Langmuir 7 , 3197-3204 (1991).

9. Brusciotti, F. \& Duby, P. Cyclic voltammetry study of arsenic in acidic solutions. Electrochim Acta 52, 6644-6649 (2007).

10. Ruan, C., Yang, L. \& Li, Y. Immunobiosensor chips for detection of escherichia coli O157:H7 using electrochemical impedance spectroscopy. Anal. Chem 74, 4814-4820 (2002).

11. Liu, G. \& Lin, Y. Electrochmical sensor for orgnohposphate pesticides and nerve agents using zirconia nanoparticles as selective sorbents. Anal. Chem 77, 5894-5901 (2005).

12. Ascaso, J. F. et al. Diagnosing insulin resistance by simple quantitative methods in subjects with normal glucose metabolism. Diabetes Care 26, 12, 3320-3325 (2003)

13. Karl, T. et al. Human breath isoprene and its relation to blood cholesterol levels: new measurements and modeling. J. Appl. Physiol 91, 762-770 (2001).

14. Armstrong, F. A. Recent developments in dynamic electrochemical studies of adsorbed enzymes and their active sites. Chem. Biol 9, 110-117 (2005).

15. Mollah, M. Y., Schennach, R., Parga, J. R. \& Cocke, D. L. Electrocoagulation (EC)science and applications. J. Hazard. Mater B84, 29-41 (2001).

16. Park, H. et al. Fully roll-to-roll gravure printed rectenna on plastic foils for wireless power transmission at $13.56 \mathrm{MHz}$. Nanotech 23, 344006-344012 (2012).

17. Kang, H. et al. Fully Roll-to-Roll Gravure Printable Wireless (13.56 MHz) SensorSignage Tags for Smart Packaging. Sci. Rep. 4, 5387; DOI: 10.1038/srep05387 (2014).

18. Jung, M. et al. All-printed and roll-to-roll-printable $13.56 \mathrm{MHz}$-operated 1-bit RF tag on plastic foils. IEEE Trans Electron Devices 57, 3, 571-580 (2010).

19. Noh, J. et al. Integrable single walled carbon nanotube (SWNT) network based thin film transistors using roll-to-roll gravure and inkjet. Org. Electron 12, 2185-5191 (2011).

20. Noh, J. et al. Modeling of printed single walled carbon nanotube thin film transistors for attaining optimized clock signals. J. Appl. Phys 108, 10, 102811-102817 (2010).

21. Noh, J. et al. Fully Gravure-Printed Flexible Full Adder Using SWNT-Based TFTs. IEEE. Eletron. Dev. Lett. 33, 11, 1574-1576 (2012).

\section{Acknowledgments}

The authors would like to thank BK Plus program, Basic Science Research program (NRF-2014R1A1030419), and RIC in Sunchon National University for their financial support. G. C. thanks to Paru Co. for providing all electronic inks in this work.

\section{Author contributions}

Y.J., A.J. and G.C. designed the experiments. Y.J., H.P., J.P., Y.C. and M.J. carried out the experiments. J.N., K.J. and K.C. performed device simulations. Y.J. and K.C. performed mobility calculations. Y.J. and M.P. performed electrochemistry and analyzed electrochemical data. Y.J., H.P., M.P., A.J. and G.C. contributed to analyzing the data. M.J., M.P., A.J. and G.C. wrote the paper while all authors provided feedback.

\section{Additional information}

Supplementary information accompanies this paper at http://www.nature.com/ scientificreports

Competing financial interests: Prof. Cho was a consultant of PARU Co. Korea and received compensation from PARU Co. Both Dr. M. Jung and Mr. K Jung has been employee of PARU Co. Korea. Dr. Y. Jung, Miss H. Park, Ms. J. Park, Dr. J. Noh, Mr. Y. Choi, Prof. Pyo, Mr. K. Chen and Prof. Javey declare no competing financial interests.

How to cite this article: Jung, Y. et al. Fully printed flexible and disposable wireless cyclic voltammetry tag. Sci. Rep. 5, 8105; DOI:10.1038/srep08105 (2015).

This work is licensed under a Creative Commons Attribution 4.0 International License. The images or other third party material in this article are included in the article's Creative Commons license, unless indicated otherwise in the credit line; if the material is not included under the Creative Commons license, users will need to obtain permission from the license holder in order to reproduce the material. To view a copy of this license, visit http://creativecommons.org/licenses/by/4.0/ 